\title{
Bruxismo na infância: um sinal de alerta para odontopediatras e pediatras
}

\author{
Childhood bruxism: a warning sign to pediatric dentists and pediatricians
}

\author{
Michele Baffi Diniz', Renata Cristiane da Silva², Angela Cristina C. Zuanon ${ }^{3}$
}

\section{RESUMO}

Objetivo: Apresentar uma revisão de literatura sobre o bruxismo na infância, abordando os fatores etiológicos, as características clínicas, os sinais e sintomas, a importância do diagnóstico por parte dos pediatras e odontopediatras e o tratamento multidisciplinar desta condição parafuncional.

Fontes de dados: Foram selecionados os artigos mais relevantes sobre o tema publicados desde 1907 até 2007, com pesquisa realizada no Medline, na Bibliografia Brasileira de Odontologia (BBO) e em livros de Odontologia.

Síntese dos dados: O bruxismo é definido como um hábito não funcional do sistema mastigatório, caracterizado pelo ato de ranger ou apertar os dentes, podendo ocorrer durante o dia e durante o sono. A etiologia é multifatorial e a literatura sugere vários fatores associados: dentário, fisiológico, psicológico e neurológico. As forças exercidas pelo bruxismo podem provocar distúrbios em diferentes graus nos dentes e nos tecidos de suporte, na musculatura e na articulação têmporo-mandibular. O sinal mais comum é o desgaste nas faces incisais dos dentes anteriores e oclusais nos posteriores, além de mobilidade e hipersensibilidade dentárias, fratura de cúspides e restaurações e hipertonicidade dos músculos mastigatórios.

Conclusão: $\mathrm{O}$ conhecimento dos fatores etiológicos e das características clínicas do bruxismo na infância é fundamental para que o diagnóstico seja precoce, permitindo que pediatras, odontopediatras e psicólogos possam estabelecer um tratamento multidisciplinar e favoreçam o desenvolvimento integral da criança para a promoção de saúde e bem-estar individual.
Palavras-chave: bruxismo; crianças; odontopediatria; Pediatria.

\section{ABSTRACT}

Objective: To present a review of literature on childhood bruxism, including etiological factors, clinical characteristics, signs and symptoms, the importance of the diagnosis by pediatric dentists and pediatricians and the multidisciplinary management of this parafunctional condition.

Data source: The most relevant articles published on the subject from 1907 to 2007 were selected, from Medline and Brazilian Bibliography on Odontology databases as well as dentistry books.

Data synthesis: Bruxism is defined as a non-functional habit of the stomatognatic system, characterized by the act of grinding or squeezing the teeth, with occurrences during the day or sleep. The etiology is multifactorial with contribution of several factors (dental, physiological, psychological and neurological variables). The forces produced by bruxism can harm the teeth, their supporting tissues, muscles and temporomandibular articulation. Common clinical signs are: attrition on the incisal faces of the anterior teeth and occlusal faces in the posterior teeth, as well as dental hypermobility and hypersensibility, cusp and restoration fracture and muscle hypertonicity.

Conclusions: The knowledge on the etiological factors and the clinical characteristics of childhood bruxism is important to establish early diagnosis and treatment, with a multidisciplinary approach that should include pediatri-
Instituição: Faculdade de Odontologia de Araraquara da Universidade Estadual Paulista "Júlio Mesquita Filho" (Unesp), Araraquara, SP, Brasil 'Mestre em Odontopediatria e aluna de Doutorado do Curso de PósGraduação em Ciências Odontológicas da Faculdade de Odontologia de Araraquara da Unesp, Araraquara, SP, Brasil

2Professora doutora da Disciplina de Saúde Coletiva da Faculdade de Odontologia da Universidade Camilo Castelo Branco, São Paulo, SP, Brasil

${ }^{3}$ Professora doutora do Departamento de Clínica Infantil da Disciplina de Odontopediatria da Faculdade de Odontologia de Araraquara da Unesp, Araraquara, SP, Brasil

\section{Endereço para correspondência: \\ Michele Baffi Diniz \\ Rua Orsini Dias Aguiar, 265 - Jardim Alvorada CEP 15020-070 - São José do Rio Preto/SP \\ E-mail: mibdiniz@hotmail.com}

Recebido em: 6/10/08

Aprovado em: 19/12/08 
cians, pediatric dentists and psychologists in order to provide adequate infant development focused on health promotion and individual well-being.

Key-words: bruxism; children; pediatric dentistry; Pediatrics.

\section{Introdução}

O bruxismo é uma atividade involuntária parafuncional, rítmica e espasmódica do sistema mastigatório produzida por contrações rítmicas ou tônicas do masseter e de outros músculos mandibulares ${ }^{(1)}$ e caracterizada pelo ato de ranger ou apertar os dentes ${ }^{(2,3)}$ tanto durante o período diurno como noturno ${ }^{(4,5)}$. De acordo com Gusson ${ }^{(6)}$, o bruxismo pode ocorrer de forma consciente, quando se mordem lápis ou dedos, ou inconscientemente. $\mathrm{O}$ ato de ranger ocorre frequentemente durante o sono, períodos de preocupação, estresse e excitação, acompanhado por um ruído notável ${ }^{(1)}$. Já o apertamento, em geral sem ruídos, é mais comum durante o $\mathrm{dia}^{(7-9)}$ e pode ser considerado mais destrutivo, uma vez que as forças são contínuas e menos toleradas ${ }^{(9)}$.

Vários fatores etiológicos podem estar associados ao desenvolvimento do bruxismo, como os fatores locais, sistêmicos, psicológicos, ocupacionais e hereditários ${ }^{(6,10,1)}$. Os sinais e sintomas mais frequentes são os desgastes oclusais e/ou incisais, destruição das estruturas de suporte, hipersensibilidade pulpar, mobilidade dentária, fratura de cúspides e restaurações, dores e distúrbios nas articulações temporomandibulares (ATM), hipertofia do masséter, cefaleia ao acordar, entre outros ${ }^{(2,12,13)}$. O bruxismo pode também estar associado a parasomnias (fenômenos que ocorrem exclusivamente durante o sono), caracterizando-se por graus diferentes de excitação, como enurese noturna, falas durante o sono e sono agitado ${ }^{(6,14)}$.

Na clínica odontológica, é comum observar desgaste excessivo nas faces oclusais e incisais das superfícies dentárias, principalmente na dentição decídua ${ }^{(15)}$. Durante a infância, o bruxismo é mais severo nas crianças em idade pré-escolar devido às características estruturais e funcionais dos dentes decíduos, embora também apareça em crianças maiores e na dentição permanente ${ }^{(15)}$.

O tratamento consiste em um trabalho multidisciplinar que abrange a odontologia, a medicina e a psicologia ${ }^{(15)}$. A odontologia normalmente atua em procedimentos restauradores, tratamento ortodôntico e placas de mordida ${ }^{(16,17)}$. Em algumas situações, pode haver a necessidade de um tratamento sistêmico com uso de medicação e tratamento médico, além de aconselhamento psicológico ${ }^{(18,19)}$.

A origem do termo bruxismo data de 1907, quando Marie Pietkiewicz ${ }^{(20)}$ utilizou a expressão la bruxomanie (bruxomania), derivada da palavra grega brychein, cujo significado é triturar ou ranger os dentes, e da palavra mania, que significa compulsão. Posteriormente, a palavra foi adaptada e, atualmente, é conhecida como bruxismo ${ }^{(1,21)}$.

Assim, o presente artigo tem como objetivo abordar a prevalência, os fatores etiológicos, as características clínicas, a sintomatologia, as medidas terapêuticas e a importância do diagnóstico precoce do bruxismo em crianças tanto por parte dos odontopediatras como pediatras, para que se possa atuar precocemente sobre essa disfunção.

\section{Importância e características clínicas}

Estudos relatam uma prevalência em torno de 7 a $20 \% \%^{(22-29)}$. Entretanto, Garcia et a ${ }^{(8)}$ relataram uma prevalência de $40 \%$ em pré-escolares de três a seis anos de idade, de $17 \%$ na faixa de seis a sete anos e $24 \%$ na faixa dos oito aos nove anos. O bruxismo esteve presente em $40 \%$ das crianças examinadas, sendo que destas, $60 \%$ apresentavam o hábito de ranger os dentes, $8 \%$ apenas apertavam e $32 \%$ rangiam e apertavam os dentes simultaneamente.

Shinkai et $a l^{(9)}$ observaram alta prevalência do bruxismo em crianças: $43 \%$ entre crianças com idade de dois a três anos, $35 \%$ entre quatro e cinco anos e $34 \%$ entre dez e 11 anos de idade. De acordo com os autores, a maioria das crianças apresentava comportamento ansioso ou hiperativo.

Em bebês, o bruxismo pode ser observado logo após a erupção dos incisivos decíduos por volta de um ano de idade $^{(30)}$. Em um estudo recentemente realizado por Petit et $\mathrm{al}^{(31)}$, observou-se que $46 \%$ das crianças examinadas com idade entre 2,5 e seis anos apresentavam bruxismo. Além disso, notou-se que a prevalência do bruxismo aumenta significativamente com a idade. Os autores observaram também que o bruxismo noturno persistente se relacionava à permanência dos pais junto aos filhos até que adormecessem.

De acordo com Bayardo et al ${ }^{(25)}$ e Hublin et al ${ }^{(32)}$, o bruxismo é mais frequente no sexo feminino, mas Barthi et al ${ }^{(29)}$ observaram que $92 \%$ das crianças com bruxismo eram do sexo masculino. Por outro lado, Demir $e t a^{(28)}$ e Petit $e t$ all ${ }^{(31)}$ não notaram diferença estatisticamente significativa na prevalência de bruxismo entre crianças de ambos os sexos. 
O bruxismo apresenta incidência de $60 \%$ em crianças alérgicas, isto é, três vezes maior do que entre crianças não alérgicas ${ }^{(33)}$. Para o autor, hábitos orais, como morder ou mascar brinquedos e lápis, sucção digital, língua protruída e respiração bucal também podem estar associados ao bruxismo.

Várias teorias sobre os possíveis fatores etiológicos relacionados ao bruxismo têm sido relatadas. Muitos acreditam que o bruxismo apresenta etiologia multifatorial ${ }^{(18,34,35)}$ que pode ser explicada por fatores locais, sistêmicos, psicológicos, ocupacionais e hereditários ${ }^{(6,9,10,15)}$.

Dentre os fatores locais, pôde-se observar maloclusões, traumatismo oclusal, contato prematuro, reabsorção radicular, presença de cálculo dental, cistos dentígeros, dentes perdidos, excesso de material restaurador e tensão muscular ${ }^{(11,36)}$. Entretanto, alguns autores observaram que a presença de maloclusão não aumenta a probabilidade de a criança desenvolver bruxismo ${ }^{(5,28)}$. Há evidências de que o bruxismo em crianças pequenas pode ser consequência da imaturidade do sistema mastigatório neuromuscular ${ }^{(17)}$. Outros autores associam a presença de bruxismo ao tempo de aleitamento materno. Em estudo realizado com 427 crianças de idade entre três e seis anos de idade, Ferreira e Toledo ${ }^{(37)}$ observaram que quanto mais prolongado o aleitamento materno, menor a ocorrência de hábitos orais nocivos, como o bruxismo.

Sistemicamente, deficiências nutricionais e vitamínicas, alergias, parasitoses intestinais, distúrbios otorrinolaringológicos, distúrbios gastrintestinais, desordens endócrinas, paralisia cerebral, Síndrome de Down e deficiência mental podem estar relacionados ao desenvolvimento do hábito ${ }^{(9,10,17,18)}$. DiFrancesco et al ${ }^{(38)}$ observaram uma associação positiva entre problemas respiratórios durante o sono, como a obstrução das vias aéreas devido à hiperplasia tonsilar, e a presença de bruxismo em crianças. Os autores relataram que, após a cirurgia de adenoides e tonsilas, as crianças apresentaram uma melhora significativa no quadro de bruxismo.

Forte tensão emocional, problemas familiares, crises existenciais, estado de ansiedade, depressão, medo e hostilidade, crianças em fase de autoafirmação, provas escolares ou mesmo a prática de esportes competitivos e campeonatos podem atuar como fatores de origem psicológica e ocupacional para o desencadeamento desta condição ${ }^{(2,15,17,21,39)}$. De acordo com Restrepo et a ${ }^{(35)}$, a ansiedade tem sido o fator emocional mais estudado em crianças.

Segundo $\mathrm{Wolf}^{\left({ }^{40)}\right.}$, o bruxismo é considerado uma resposta de escape, uma vez que a cavidade bucal possui um forte potencial afetivo, além de ser um local privilegiado para a expressão de impulsos reprimidos, emoções e conflitos. Dessa forma, algumas crianças, por não conseguirem satisfazer seus anseios, desejos e necessidades, acabam por ranger ou apertar os dentes para compensar tais problemas ou como uma forma de autoagressão.

Vanderas ${ }^{(5)}$ observou aumento de atividade parafuncional em um grupo de crianças tensas, ansiosas, nervosas ou sob estresse. Shinkai $e t a^{(9)}$ relataram que o comportamento diário da criança atua de maneira significativa na ocorrência do bruxismo em comparação a situações passageiras de estresse emocional. As variações na personalidade da criança muitas vezes são responsáveis pelo desenvolvimento deste hábito ${ }^{(41)}$.

Com relação aos fatores hereditários, um estudo sobre predisposição genética confirmou que pais que possuíam o hábito na infância frequentemente apresentam filhos que apertam ou rangem os dentes ${ }^{(22)}$. Reding et a $l^{(7)}$ e Glaros ${ }^{(42)}$ também observaram que crianças de pais com bruxismo são mais suscetíveis ao hábito, o que sugere uma predisposição hereditária, embora o modo de transmissão ainda seja desconhecido.

Weideman et al ${ }^{(14)}$ verificaram que alguns fatores relacionados ao comportamento e condições físicas (por exemplo, mioespasmo muscular, enurese noturna, cólica, excessiva salivação noturna e conversar dormindo) apresentaram diferença significativa entre pacientes com e sem o hábito. Desta forma, os autores sugerem a possibilidade de que o bruxismo e outras parasomnias possam ocorrer simultaneamente em crianças predispostas a tais distúrbios do sono.

Mais recentemente, um estudo relatou o comportamento de neurotransmissores, principalmente a L-dopamina, no desenvolvimento de bruxismo. Observou-se, em pacientes com bruxismo noturno, que a dopamina não exacerba o hábito e, ao contrário, reduz sua frequência em aproximadamente $30 \%{ }^{(34)}$. Entretanto, novos estudos clínicos são necessários para que se estabeleça uma relação entre as substâncias adrenérgicas e o bruxismo, uma vez que o efeito pode ser tanto central à excitabilidade do sistema motor, como periférico, induzindo relaxamento ${ }^{(1)}$.

Segundo Gusson ${ }^{(6)}$, a criança pode desenvolver bruxismo após a erupção dos incisivos centrais decíduos, ocasionando dilacerações gengivais nos casos em que o antagonista ainda não erupcionou. $\mathrm{O}$ bruxismo infantil pode ser caracterizado pela presença de desgastes da superfície dentária, desconfortos musculares e articulares, atuando como coadjuvante na progressão da doença periodontal destrutiva e contribuindo para o desenvolvimento de falsa Classe III, além de acelerar a rizólise de dentes decíduos e provocar alterações na cronologia de erupção dos dentes permanentes. Descreve-se, também, a possibilidade de o bruxismo favorecer o apinhamento dental ${ }^{(11,18)}$. 
As facetas de desgaste observadas no bruxismo podem ser lisas (devido ao rangimento) ou rugosas (apertamento), de bordos bem definidos na fase inicial, atingindo mais os dentes anteriores do que os posteriores, principalmente caninos decíduos e permanentes ${ }^{(8,17)}$. A presença de facetas de desgaste muitas vezes indica história pregressa de bruxismo, que pode não estar mais ocorrendo no momento do exame clínico ou estar se iniciando ${ }^{(43)}$.

Os efeitos do bruxismo nos dentes dependem de vários fatores, como o tipo e gravidade da parafunção, número e localização dos dentes, posicionamento dentário na arcada, relações intermaxilares, altura das cúspides, mobilidade e contato interdentário ${ }^{(44,45)}$.

Como o bruxismo é um reflexo subconsciente não controlado e leve, na maioria das vezes é desconhecido ou despercebido pelos pacientes e seus familiares. Assim, torna-se necessário estabelecer o diagnóstico precoce das possíveis alterações que poderão ocorrer, antes que o ciclo vicioso resulte em dano grave e permanente ${ }^{(4)}$.

Em crianças, essa desordem normalmente é identificada pelos odontopediatras diante de desgastes dentários anormais, disfunções temporomandibulares e dor. No entanto, os pediatras podem reconhecer essa desordem em pacientes que procuram atendimento por dores de cabeça atípicas ou dores mandibulares ao acordar ${ }^{(1)}$. Liljestrom et a ${ }^{(46)}$ verificaram que tanto o bruxismo como as desordens temporomandibulares são encontrados em crianças que apresentam episódios de dores de cabeça.

A sintomatologia dolorosa depende da frequência, intensidade e idade do paciente, em associação à duração do hábito. Nas crianças, os sinais e sintomas são mais ocasionais e leves, o que explica o fato de elas estarem, na maioria das vezes, livres da disfunção da articulação temporomandibular ${ }^{(18)}$.

É de fundamental importância realizar a anamnese completa da criança em ambiente tranquilo, contando com a participação dos pais para obter informações sobre a história médica geral, hábitos, queixa de dor, relacionamento familiar e social e avaliação do perfil psicológico da criança. $\mathrm{O}$ exame clínico minucioso, com palpação, ausculta, avaliação de tecidos moles e da língua, verificação da movimentação mandibular, análise da oclusão, bom como os exames radiográficos são importantes no diagnóstico preciso das alterações do sistema estomatognático ${ }^{(10)}$.

A avaliação do desgaste dentário em pacientes com bruxismo se baseia principalmente no diagnóstico visual, sem diferenciar se a causa está relacionada a fatores psicológicos ou patológicos ${ }^{(47)}$. Segundo Restrepo et al ${ }^{(48)}$, imagens digitalizadas do desgaste dentário podem ser utilizadas como critério diagnóstico de bruxismo em crianças na dentição mista, após análise da área, perímetro e irregularidade na forma do desgaste dentário, diferenciando-se o desgaste patológico do psicológico.

Embora poucos estudos tenham analisado o desenvolvimento natural do bruxismo noturno, esta parece ser uma doença crônica persistente ${ }^{(1)}$. De acordo com Abe e Shimakawa ${ }^{(22)}$, o bruxismo, quando identificado na infância, persiste em $35 \%$ dos pacientes até a vida adulta. Mais recentemente, Hublin et a ${ }^{(32)}$ relataram que os sintomas do bruxismo podem persistir em $86 \%$ dos casos na fase adulta.

É de fundamental importância o diagnóstico diferencial para que o bruxismo não seja confundido com outros movimentos faciais do sono, tais como atos de engolir, tossir, grunhir ou alternar a abertura e o fechamento mandibulares ${ }^{(49)}$. Além disso, é necessário distinguir o ruído proveniente do bruxismo daquele causado pelo ato de roncar por meio de gravações ou técnicas de vigilância, uma vez que o ruído desencadeado pelo ranger dos dentes é característico em pacientes bruxistas ${ }^{(1)}$.

\section{A importância do tratamento multidisciplinar}

A principal intervenção clínica relacionada ao bruxismo deve ser voltada para a proteção do dente, reduzindo o ranger, aliviando dores faciais e temporais e promovendo melhorias na qualidade do sono, caso seja deficiente. Para tanto, três tipos de estratégias devem ser empregadas: dentária, farmacológica e psico-comportamental ${ }^{(1)}$.

Como a etiologia do bruxismo é, na maioria das vezes, um conjunto de fatores, o tratamento psicológico é importante, principalmente quando iniciado com o uso da placa de mordida. Essa placa visa a reduzir a atividade parafuncional, desprogramar e induzir ao relaxamento muscular, obter uma proteção dos dentes contra a atrição e desgaste, balanceio dos contatos oclusais, bem como reposicionar a mandíbula, colocando-a em uma relação normal com a maxila para alcançar um equilíbrio neuromuscular ${ }^{(1,16,50)}$. A placa de mordida tem como vantagem não interferir no processo de crescimento das arcadas dentárias infantis ou alterar as suas características físicas, além de ser um tratamento reversível, de boa aceitação pelas crianças e eficaz ${ }^{(16-18)}$. De acordo com Solberg et $a^{(51)}$, o uso de placa de mordida reduz a atividade muscular, proporcionando maior conforto ao paciente. O material de escolha para a confecção da placa deve ser à base de silicone, com espessura de aproximadamente $3 \mathrm{~mm}$, estendendo-se da região 
vestibular à lingual o suficiente para prevenir a perfuração e aumentar a resistência ao impacto ${ }^{(52)}$.

Considerando, ainda, o tratamento dentário, deve-se avaliar a necessidade de ajustar a oclusão do paciente e restaurar as superfícies dentárias e contornos com materiais adequados e indicados para cada caso. Em alguns pacientes, o tratamento ortodôntico se faz necessário. Essas intervenções são extensas e irreversíveis e, consequentemente, não são indicadas na maioria dos $\operatorname{casos}^{(53,54)}$.

Considerando a abordagem psico-comportamental, Ahmad $^{(18)}$, Attanasio ${ }^{(2)}$ e Bader e Lavigne ${ }^{(1)}$ indicam a psicoterapia (aconselhamento, autossugestão, hipnose, condicionamento, exercícios de relaxamento e biofeedback), medicamentos, como injeção de anestésico local na região, tranquilizantes, sedativos, miorrelaxantes e placebos. O uso de métodos elétricos, como estímulos eletrogalvânicos para o relaxamento muscular, sons (ultrassônicos) ou acupressão de áreas também são indicados. $\mathrm{Na}$ ausência de fatores sistêmicos, os autores preconizam os exercícios orais para dessensibilização (sensibilidade térmica, aconselhamento nutricional, suplemento de vitaminas e minerais).

Alguns estudos têm relatado que o uso de benzodiazepínicos (diazepam), relaxantes musculares (metocarbomol), precursores de catecolaminas (L-dopa) e antagonistas betaadrenérgicos (propanolol) podem reduzir a frequência de bruxismo noturno ${ }^{(55,56)}$. Entretanto, ainda não existem estudos clínicos que comprovem a eficácias desses medicamentos na faixa etária pediátrica. Dessa forma, tais medicamentos devem ser utilizados com cautela, optando-se sempre por outras estratégias para o controle do bruxismo.

Segundo Marini ${ }^{(57)}$, antes de dar início a qualquer tipo de tratamento, os pais devem ser esclarecidos sobre a etiologia do bruxismo. De acordo com o autor, o ideal é a terapia multidisciplinar com dentista, psicólogo e o próprio paciente motivado. O tratamento proposto pelo profissional deve, portanto, abranger aspectos dentários, médicos e psicológicos, além da motivação do paciente ${ }^{(58)}$.

\section{Referências bibliográficas}

1. Bader G, Lavigne G. Sleep bruxism: an overview of an oromandibular sleep movement disorder. Sleep Med Rev 2000;4:27-43.

2. Attanasio R. Nocturnal bruxism and its clinical management. Dent Clin North Am 1991;35:245-52.

3. Pingitore G, Chrobak V, Petrie J. The social and psychologic factors of bruxism. J Prosthet Dent 1991;65:443-6.

4. Ramfjord S, Ash MM, editores. Oclusão. $3^{\mathrm{a}}$ ed. Rio de Janeiro: Guanabara
$\mathrm{Na}$ realidade não existe nenhum tratamento específico para o bruxismo. Cada paciente deve ser avaliado individualmente e tratado de acordo com os fatores possivelmente associados para que futuras complicações dentárias sejam evitadas. O tratamento escolhido para crianças com bruxismo deve sempre ser reversível e conservador, de modo a não interferir negativamente no padrão de normalidade da dinâmica de crescimento e desenvolvimento infantil ${ }^{(50)}$.

\section{Conclusões}

O bruxismo na infância é uma disfunção que vem crescendo em frequência na sociedade moderna. A carência de atendimento pode acarretar danos severos na cavidade bucal e na musculatura facial. Vários fatores predispõem uma pessoa ao desenvolvimento do bruxismo, sendo preponderantes os fatores psicológicos, como a ansiedade e o estresse emocional. O bruxismo é de difícil diagnóstico e o tratamento ideal é a terapia multidisciplinar. $\mathrm{O}$ diagnóstico precoce em crianças visa a manter a perspectiva de controle e prevenção de danos aos componentes do sistema mastigatório, além de propiciar bem-estar e conforto. O pediatra, por ser o primeiro profissional da saúde a estabelecer contato com a criança, possui importante papel no diagnóstico dessa desordem, atuando de forma efetiva no reconhecimento do problema e no encaminhamento aos demais profissionais da área de saúde, como os odontopediatras e psicólogos.

O pediatra e o odontopediatra devem estar aptos a compreender as possíveis causas, características clínicas, sinais e sintomas do bruxismo na infância, identificando o problema o mais precocemente possível. Uma vez diagnosticada a origem psicológica, é preciso encaminhar o paciente a um profissional especializado a fim de proporcionar um tratamento eficaz e duradouro. É fundamental que haja uma interação entre os profissionais das diversas especialidades envolvidas no tratamento de bruxismo em crianças, como os odontopediatras, pediatras e psicólogos, com o objetivo de acompanhar o crescimento e o desenvolvimento da criança, promovendo a saúde.

Koogan; 1987.

5. Vanderas AP, Manetas KJ. Relationship between malocclusion and bruxism in children and adolescents: a review. Pediatr Dent 1995;17:7-12.

6. Gusson DGD. Bruxismo em crianças. J Bras Odontoped Odonto Bebe 1998;1:75-97.

7. Reding GR, Rubright WC, Zimmerman SO. Incidence of bruxism. J Dent Res 1966;45:1198-204. 
8. Garcia PP, Corona SA, Santos-Pinto A, Sakima T. Verificação da incidência de bruxismo em pré-escolares. Odontol Clin 1995;5:119-22.

9. Shinkai RS, Santos LM, Silva FA, Santos MN. Prevalence of nocturnal bruxism 2-11-year-old children. Rev Odontol Univ São Paulo 1998;12:29-37.

10. Nor JE, Feldens EG, Witt SMR, Scherer SC, Thomazi TH, Martins EA et al. Bruxismo em crianças. Rev Fac Odontol 1991;32:18-21.

11. Maciel RN. Oclusão e ATM: procedimentos clínicos. São Paulo: Santos; 1996.

12. Lavinas MO. O bruxismo como manifestação bucal de distúrbios psicogênicos. Odontol Moder 1985;12:54-8.

13. Bailey DR. Tension headache and bruxism in the sleep disordered patient. Cranio 1990;8:174-82.

14. Weideman CL, Bush DL, Yan-Go FL, Clark GT, Gornbein JA. The incidence of parasomnias in child bruxers versus nonbruxers. Pediatr Dent 1996;18:456-60.

15. Alves VC, Moliterno LF, Ramos ME, Cruz RA, Campos V. Alguns aspectos do bruxismo de interesse do odontopediatra. Rev Odontopediatr 1993;2:157-63.

16. Hachmann A, Martins EA, Araujo FB, Nunes R. Efficacy of the nocturnal bite plate in the control of bruxism for 3 to 5 year old children. $\mathrm{J}$ Clin Pediatr Dent 1999:24:9-15.

17. Antonio AG, Pierro VS, Maia LC. Bruxism in children: a warning sign for psychological problems. J Can Dent Assoc 2006;72:155-60.

18. Ahmad R. Bruxism in children. J Pedod 1986;10:105-26.

19. McDonald RE, Avery DR. Odontopediatria. $6^{\mathrm{a}}$ ed. Rio de Janeiro: Guanabara Koogan; 1995.

20. Pietkiewicz M. La bruxomanie: memoires originaux. Rev Stomatol 1907;14:107-16.

21. Silva SR. Bruxismo. Rev Assoc Paul Cir Dent 2003;57:409-17.

22. Abe K, Shimakawa M. Genetic and developmental aspects of sleeptalking and teeth-grinding. Acta Paedopsychiatr 1966;33:339-44.

23. Nilner M, Kopp S. Distribution by age and sex of functional disturbances and diseases of the stomatognathic system in 7-18 year olds. Swed Dent $\mathrm{J}$ 1983;7:191-8.

24. Widmalm SE, Christiensen RL, Gunn SM. Oral parafunctions as temporomandibular disorder risk factors in children. Cranio 1995;13:242-6.

25. Bayardo RE, Mejia JJ, Orozco S, Montoya K. Etiology of oral habits. ASDC J Dent Child 1996;63:350-3.

26. Laberge L, Tremblay RE, Vitaro F, Montplaisir J. Development of parasomnias from childhood to early adolescence. Pediatrics 2000;106:67-74.

27. Campos AD, Campos AG, Zuanon AC. Bruxismo em crianças. Rev Odontopediatr 2002;50:74-6.

28. Demir A, Uysal T, Guray E, Basciftci FA. The relationship between bruxism and occlusal factors among seven- to 19-year-old Turkish children. Angle Orthod 2004;74:672-6.

29. Bharti B, Malhi P, Kashyap S. Patterns and problems of sleep in school going children. Indian Pediatr 2006;43:35-8.

30. Diagnostic Steering Committee, Thorpy JM, Chairman. ICSD: International classification of sleep disorders: diagnostic and coding manual. Rochester, Minnesota: American Sleep Disorders Association; 1990.

31. Petit D, Touchette E, Tremblay RE, Boivin M, Montplaisir J. Dyssomnias and parasomnias in early childhood. Pediatrics 2007;119:e1016-25.

32. Hublin C, Kaprio J, Partinen M, Koskenvuo M. Sleep bruxism based on selfreport in a nationwide twin cohort. J Sleep Res 1998;7:61-7.

33. Marks MB. Bruxism in allergic children. Am J Orthod 1980;77:48-59.

34. Lobbezoo F, Lavigne GJ. Do bruxism and temporomandibular disorders have a cause-and-effect relationship? J Orofac Pain 1997;11:15-23.

35. Restrepo CC, Alvarez E, Jaramillo C, Vélez C, Valencia I. Effects of psychological techniques on bruxism in children with primary teeth. J Oral
Rehabil 2001;28:354-60.

36. Meklas JF. Bruxism: diagnosis and treatment. J Acad Gen Dent 1971;19:31-6.

37. Ferreira MI, Toledo OA. Relação entre tempo de aleitamento materno e hábitos bucais. Rev ABO Nac 1997;5:317-20.

38. Di Francesco RC, Junqueira PA, Trezza PM, Faria ME, Frizzarini R, Zerati FE. Improvement of bruxism after T \& A surgery. Int J Pediatr Otorhinol 2004;68:441-5.

39. Cariola TC. O desenho da figura humana de crianças com bruxismo. Bol Psicol 2006;56:37-52.

40. Wolf SM, editor. Psicologia no consultório odontológico. São Paulo: Arte \& Ciência; 2000.

41. Glaros AG, Rao SM. Effects of bruxism: a review of the literature. J Prosthet Dent 1977;38:149-57.

42. Glaros AG. Incidence of diurnal and nocturnal bruxism. J Prosthet Dent 1981;45:545-9.

43. Vanderas AP. Relationship between craniomandibular dysfunction and oral parafunctions in Caucasian children with and without unpleasant life events. J Oral Rehabil 1995;22:289-94.

44. Kerstein R. Disclusion time measurement studies: stability of disclusion time - a 1-year follow-up. J Prosthet Dent 1994;72:164-8.

45. Mehl A, Gloger W, Kunzelmann KH, Hickel R. A new optical 3-D device for the detection of wear. J Dent Res 1997;76:1799-807.

46. Liljeström MR, Jämsä T, Le Bell Y, Alanen P, Anttila P, Metsähonkala L et al. Signs and symptoms of temporomandibular disorders in children with different types of headache. Acta Odontol Scand 2001;59:413-7.

47. Negoro T, Briggs J, Plesh O, Nielsen I, McNeill C, Miller AJ. Bruxing patterns in children compared to intercuspal clenching and chewing as assessed with dental models, electromyography, and incisor jaw tracing: preliminary study. ASDC J Dent Child 1998;65:449-58.

48. Restrepo C, Peláez A, Alvarez E, Paucar C, Abad P. Digital imaging of patterns of dental wear to diagnose bruxism in children. Int $\mathrm{J}$ Paediatr Dent 2006;16:278-85.

49. Velly-Miguel AM, Montplaisir J, Rompré PH, Lund JP, Lavigne GL. Bruxism and other orofacial movements during sleep. J Craniomand Dis Fac Oral Pain 1992;6:71-81.

50. Parizotto SPCOL, Rodrigues CRMD. Tratamento de bruxismo em crianças através do uso de placa de mordida e reabilitação das facetas de desgaste. J Bras Odontoped Odonto Bebe 1999;2:339-44.

51. Solberg WK, Clark GT, Rugh JD. Nocturnal electromyographic evaluation of bruxism patients undergoing short term splint therapy. J Oral Rehabil 1975;2:215-23.

52. Cassamassimo P. Periodontal considerations. In: Pinkham J, Cassamassimo OS, Fields HW, McTigue DJ, Nowak A, editors. Pediatric dentistry infancy through adolescence. $2^{\text {nd }}$ ed. Philadelphia: Saunders; 1994. p. 372.

53. Clark GT, Adler RC. A critical evaluation of occlusal therapy: occlusal adjustment procedures. J Am Dent Assoc 1985;110:743-50.

54. Okeson JP. Orofacial pain guidelines for assessment, diagnosis and management. Illinois: American Academy of Orofacial Pain; 1996.

55. Amir I, Hermesh H, Gavish A. Bruxism secondary to psychotic drug exposure: a positive response to propanolol. Clin Neuropharmacol 1997;20:86-9.

56. Lobbezoo F, Lavigne GJ, Tanguay R, Montplaisir J. The effect of catecholamine precursor L-dopa on sleep bruxism: a controlled clinical trial. Movement Disorders 1997;12:73-8.

57. Marini LA. Problemas comunes de oclusión en odontología pediátrica. Acta Odont Pediatr 1985;6:19-21.

58. Zuanon AC, Campos CG, Giro EM, Pansani CA. Bruxismo infantil. Odontol Clin 1999;9:41-4. 